\title{
Entrelacs
}

Cinéma et audiovisuel

\section{Les « nouvelles » formes à succès des feuilletons télévisés sud-coréens}

\section{Stéphane Thévenet}

\section{(2) OpenEdition}

\section{Journals}

Édition électronique

URL : http://journals.openedition.org/entrelacs/262

DOI : 10.4000/entrelacs.262

ISSN : 2261-5482

Éditeur

Éditions Téraèdre

Édition imprimée

Date de publication : 7 février 2008

ISSN : 1266-7188

Référence électronique

Stéphane Thévenet, "Les «nouvelles » formes à succès des feuilletons télévisés sud-coréens », Entrelacs [En ligne], HS | 2008, mis en ligne le 01 août 2012, consulté le 02 mai 2019. URL : http:// journals.openedition.org/entrelacs/262; DOI : 10.4000/entrelacs.262

Ce document a été généré automatiquement le 2 mai 2019.

Tous droits réservés 


\title{
Les « nouvelles» formes à succès des feuilletons télévisés sud-coréens
}

\author{
Stéphane Thévenet
}

1 En moins de dix ans, la Corée du Sud a su se faire une place parmi les grands producteurs audiovisuels mondiaux et se présente à l'heure actuelle comme un producteur incontournable de l'aire asiatique, supplantant le Japon. Cet engouement pour la culture populaire coréenne en Asie, appelé "Vague coréenne" (Hallyu), qui semble s'atténuer aujourd'hui, a débuté au milieu des années 90 et concerne la musique, le cinéma, mais avant tout les productions télévisées (feuilletons). L'engouement des pays voisins n'aurait cependant pas été possible sans le succès des "dramas" sur le territoire national. Depuis les débuts de la télévision, en effet, les feuilletons prédominent dans les grilles de programmes et obtiennent régulièrement des taux d'audience importants. L'introduction du magnétoscope, puis de l'Internet a ensuite fait d'eux des produits audiovisuels que l'on s'échange, et à propos desquels, on échange facilement.

\section{Une nette préférence pour le feuilleton classique}

2 En Corée, les fictions unitaires (essentiellement des téléfilms) et sérielles (certaines sitcoms) étant minoritaires, la grande majorité des fictions sont de type feuilletonesque (Benassi, 2000). D'une durée variable de 30 à 70 minutes, les feuilletons coréens sont visibles tout au long de la journée et dénommés suivant leur programmation : Dramas du matin, Dramas quotidiens, Dramas du lundi et du mardi, Dramas du mercredi et du jeudi, Dramas du vendredi, Dramas du week-end. Excepté les Dramas du matin ou les Dramas quotidiens au format plus court, les dramas sont en général diffusés le soir après le journal télévisé et durent de 50 à 70 minutes sans interruptions publicitaires.

3 Traditionnellement, les fictions télévisuelles sud-coréennes aux tons réalistes et mélodramatiques très prononcés se déclinent en trois genres principaux, à savoir, les drames historiques, les drames familiaux et les drames romantiques.

Le feuilleton historique classique, très présent sur les écrans coréens, a souvent plus de 
cinquante épisodes, diffusés deux fois par semaine. Il narre d'une façon plus ou moins romancée certains épisodes de l'histoire nationale, et particulièrement ceux situés pendant la longue dynastie Joseon (1392-1910) ainsi qu'aux époques charnières où se sont illustrés les héros fondateurs. Des scènes de combats y alternent avec d'autres, d'entretiens officiels, de préparation de plans de bataille, de complots... L'accent est généralement mis sur la beauté des décors et des costumes. Ce genre est très apprécié du public, notamment masculin. Récemment Prince of the Legend, (Titre original : Jumong) un feuilleton de 81 épisodes a réussi à rassembler 50 \% de l'audience lors du dernier épisode. Même s'ils restent des fictions à part entière, les feuilletons historiques, en offrant aux téléspectateurs des informations sur la période mise en scène, prennent parfois l'allure d'un docu-fiction (Hong-Mercier, 2006). Ces informations, données en sous-titre, sont de nature très diverse. Il peut s'agir simplement de dates de règne d'un roi mais aussi, par exemple, de recettes anciennes, d'explications sur des termes et des expressions archaïques... Il n'est pas rare alors que les caractères chinois, autrefois très usités et plus explicites, viennent compléter le sous-titre.

Dans le drama familial, le téléspectateur retrouvait souvent la famille traditionnelle coréenne, c'est-à-dire une famille élargie où trois générations habitaient sous le même toit. Le format propice était alors le feuilleton quotidien. Certains d'entre eux ont eu une longévité impressionnante (Journal de campagne a duré plus de 22 ans). Désormais, le drama familial donne plus souvent à voir une famille réduite à un couple avec un ou deux enfants en bas âge à qui les beaux-parents rendent visite régulièrement ou bien avec qui habite un des beaux-parents encore vivant. Ces feuilletons fidèles au genre, traitent des problèmes et des conflits au sein de la famille (infidélité, divorce, mésentente avec les beaux-parents, difficultés économiques, secrets de famille, graves maladies).

Les drames sentimentaux sont les plus nombreux. Pendant longtemps, les scènes de demande et de cérémonie de mariage ont constitué des passages obligés. Aujourd'hui cette nécessité sociale est moins mise en avant mais les scénaristes réussissent toujours à empêtrer les amoureux dans diverses intrigues personnelles, familiales ou professionnelles, qui retardent ou compliquent ce moment. Les triangles amoureux (voire "carré" amoureux !) sont favorisés par la retenue dans la relation amoureuse, dont sont censés faire preuve les protagonistes, et notamment les jeunes filles cantonnées pendant longtemps dans des rôles de jeunes filles belles et naïves découvrant leur premier amour. En fait, le téléspectateur coréen mais aussi asiatique, retrouve en général les valeurs qui empreignent bon nombre de récits auxquels il est habitué : amour, sens de la famille, sens de l'honneur, recherche du mérite, fidélité, amitié. Si l'on ajoute la fatalité (maladie, accident) et les questions d'identité (notamment paternité cachée, sœur ou frère ignoré...) qui abondent, nous avons là tous les éléments du mélodrame classique.

\section{Nouvelles formes et nouvelles tendances}

6 Ces trois genres subsistent aujourd'hui mais beaucoup de feuilletons deviennent plus difficilement classables, tant les nouvelles formes créées bouleversent les habitudes. En particulier, les formes du trendy drama et du fusion drama, telles que les appellent les spécialistes des médias asiatiques, apparues à la télévision sud-coréenne au début des années 90, semblent devenir la norme. Formes modernes des drames familiaux et romantiques pour l'un, des drames historiques pour l'autre, elles sont plébiscitées 
aujourd'hui non seulement par les téléspectateurs sud-coréens mais aussi par les téléspectateurs asiatiques.

Le trendy drama est en fait né au Japon dans les années 80 (Clements \& Tamamuro, 2004). Destiné à la nouvelle classe des jeunes consommateurs japonais, il est vite devenu populaire, a été exporté massivement en Asie dans les années 80 jusqu'au début des années 90 (l'Asie connaissait alors une "Vague japonaise") et est devenu le modèle à suivre dans de nombreux pays, dont la Corée du Sud, où les productions télévisuelles ont été pendant longtemps, fortement influencées par le Japon.

Les principales caractéristiques des trendy dramas sont un environnement en grande partie urbain, une majorité de personnages jeunes, célibataires ou nouvellement mariés, aux professions modernes telles que designer, architecte, présentateur de télévision, vedettes, mannequins... Avant tout, les trendy dramas développent surtout leur intrigue dans un temps proche du celui du téléspectateur. Cette contemporanéité facilite ainsi l'introduction de produits placés (notamment téléphone, automobile, vêtements, meubles) et la promotion de lieux de tournages (restaurants, centre commerciaux, complexes de cinéma, parcs de loisirs, parcs nationaux, plages...). En fait, même si les problèmes de société sont rarement traités et affrontés directement, ces feuilletons sont très ancrés dans la quotidienneté des téléspectateurs et reflètent certaines tendances de la société. Partageant de nombreux référents (rôles principaux interprétés par les stars du moment, bandes originales que l'on retrouve partout dans la rue et sur les ondes, produits placés, lieux communs de la ville) avec le téléspectateur et l'internaute, ils sont emblématiques de la société coréenne contemporaine, faisant de lui un produit de consommation courante. Le goût des Coréens pour la nouveauté, la modernité, les rend très profitables pour les compagnies de télévision et de publicités. Il est intéressant à ce propos de noter les habitudes de consommation du drama coréen : la grande majorité des téléspectateurs et internautes le regarde généralement pendant ou peu après sa période de diffusion à la télévision. Les seules rediffusions proposées sur le petit écran sont proposées dans la même semaine, pendant la journée ou le week-end suivant.

8 Le fusion drama, quant à lui, est en fait un feuilleton historique d'une durée plus réduite et à la forme et au ton très modernes. Il se permet des audaces en termes de mode de filmage, d'angles de prises de vue et d'effets techniques. Il attire ainsi des téléspectateurs plus jeunes qui ne regarderaient peut-être pas de feuilletons historiques. Ces dernières années, les fusions dramas se sont faits nombreux sur les petits écrans coréens. Deux ont eu particulièrement du succès: Jewel in the Palace (Dae-jang-geum, MBC, 2003-2004, 54 épisodes) et The Legendary Police Woman (Damo, MBC, 2003, 14 épisodes). Le premier diffusé en Corée en 2003 et 2004 raconte les aventures d'une jeune femme orpheline, qui, des cuisines du palais où elle est apprentie, réussit à devenir médecin personnel du roi. Ce feuilleton a obtenu un taux d'audience moyen de $45,8 \%$ en Corée et a été vendu à de nombreux pays.

Le feuilleton The Legendary Police Woman (Damo), quant à lui, n'a pas eu autant d'audience télévisée mais en revanche a vu se constituer autour de son nom de nombreux fandoms. L'action a beau se passer au $17^{\text {ème }}$ siècle, la réalisation est très moderne. Damo désignait les femmes de basse classe qui avaient pour première tâche de faire et de servir le thé chez les aristocrates mais étaient aussi employées pour mener des enquêtes auprès de femmes aristocrates difficilement approchables par des hommes. Le feuilleton raconte la vie d'une de ces policières d'une autre époque, particulièrement intelligente et courageuse. Bien que son taux d'audience ait été moyen, la façon de filmer 
particulièrement moderne, les différents effets spéciaux (ralentis, accélérés, zooms sur des détails comme les parties du corps des victimes examinées par la police "scientifique" de cette époque) qui rappellent la série américaine CSI: les Experts, l'alternance de musiques rock et ballade, l'utilisation d'un langage moins archaïque et de nombreux autres éléments ont plu aux jeunes téléspectateurs.

\section{Nouvelles tendances : esthétique, fantaisie et originalité}

9 Il semblerait que le développement de la forme du fusion drama soit à mettre en relation avec l'avènement de celle du trendy drama car elle partage avec cette dernière ses principales caractéristiques et notamment l'interprétation des rôles principaux par des acteurs et actrices jeunes et beaux, une bande originale moderne soignée, de beaux paysages, et l'ajout de scènes comiques. Les téléspectateurs coréens attachent en effet une grande importance à la beauté des acteurs qui interprètent les héros de leurs fictions préférées ainsi qu'à la musique, très présente, qui accompagne leurs émotions devant l'écran. De gros efforts esthétiques ont donc été réalisés tout au long de la dernière décennie. Un soin particulier a aussi été apporté dans la mise en scène des paysages, naturels ou non (nombreux tournages à l'étranger). Ainsi, certaines séquences s'apparentent à des clip-vidéos, en particulier les plus sentimentales d'entre elles. L'innovation technologique est au service de ces exigences esthétiques. Les dramas coréens offrent ainsi, en général, une qualité d'image et de sons avancée : quelques-uns des plus récents sont produits selon la norme HD (ex: Princess Hours, The Legendary Police Woman).

Le ton mélodramatique, privilégié pendant des décennies, l'est de moins en moins et plusieurs feuilletons présentent des scènes dignes de comédies de situation. Autrefois réservées aux personnages secondaires, ces scènes sont dorénavant le fait des personnages principaux dans les trendy dramas.

Les scénaristes de fusion dramas, de leur côté, pour échapper à l'austérité du genre historique, ont su inventer des rôles secondaires à des fins humoristiques.

L'innovation vient également du côté de l'énonciation. Certains dramas alternent ainsi les points de vue comme dans My name is Kim Sam-sun, un succès de 2005, où le téléspectateur se retrouve projeté parfois dans les scènes imaginées par Sam-sun, l'héroïne, opposé de l'archétype du personnage principal féminin.

La recherche d'originalité, de fantaisie a conduit les producteurs à se tourner vers de nouvelles sources comme la bande dessinée. Ainsi, dans Princess Hours (Titre original: Gung = Le palais), il a été imaginé que la Corée actuelle était une monarchie constitutionnelle où le roi régnant malade poussait son jeune héritier à se marier contre son gré avec une fille du peuple suite à un accord secret. Lors des premiers épisodes, à partir du moment où la jeune fille accepte de rencontrer la famille puis de s'installer au palais, il y a ainsi un clin d'oeil amusant: alors que dans les drames historiques, les termes archaïques sont fréquemment expliqués aux téléspectateurs, dans Princess Hours, tout au moins au début pendant la période d'adaptation, c'est la jeune fille qui, usant de termes argotiques connus des jeunes Coréens d'aujourd'hui, n'est pas comprise de la reine mère et ce sont ses paroles à elle, qui sont expliquées en surimpression à l'écran par divers procédés rappelant les bulles de bande dessinées. Les inscriptions graphiques, couramment utilisées par ailleurs dans ce feuilleton, nous rappellent la base du scénario. 


\section{Le règne de la mini-série : peu de prouesses narratologiques}

10 L'évolution vers les formes modernes présentées plus haut nécessitant un investissement considérable, a contribué au raccourcissement général des fictions. Le format de mini-série (en fait "mini-feuilleton") s'est imposé dans les années 90 et aujourd'hui les seuls feuilletons à dépasser la centaine d'épisodes sont les feuilletons quotidiens diffusés en matinée ou en tout début de soirée avant le journal télévisé, ainsi que les feuilletons historiques de forme classique. Les feuilletons de forme trendy ou fusion, toujours diffusés dans la soirée sont en fait des mini-séries dont le nombre d'épisodes ne dépasse que très rarement 20 épisodes pour les premiers et 70 épisodes pour les seconds.

Une vingtaine d'épisodes ne permettant pas vraiment de développer une grande quantité de fils narratifs, le système narratif des trendy dramas repose principalement sur une intrigue principale à laquelle se greffent des intrigues secondaires. En revanche, les analepses et les prolepses ont fréquemment été utilisées pour accentuer le côté dramatique. Beaucoup de dramas se déroulaient ainsi en trois temps: l'enfance ou l'adolescence, périodes pendant lesquelles les personnages se rencontraient, le début de l'âge adulte au moment où l'on prend des engagements et quelques années après, une fois que les tensions étaient retombées. L'usage fait de ces deux procédés dans les fictions nouvelles semble plus réduit mais cependant plus élaboré. Il faut aussi noter qu'outre le saut dans le temps, "le saut dans l'espace", entendez par là une localisation d'un ou plusieurs épisodes à l'étranger, a également servi de ressort dramatique et cela essentiellement au tout début des années 2000 à l'apogée de la Vague coréenne.

\section{Conclusion}

11 L'originalité des mini-séries sud-coréennes, construites autour de matrices similaires, vient en fait du ton et de la forme. En effet, bien que perdurent les accents mélodramatiques dans beaucoup d'entre eux, les feuilletons de ces dernières années ont su s'enrichir de situations plus légères, voire comiques et se rapprochent des comédies de situations. La violence est beaucoup moins présente et alors que les personnages masculins se rapprochent plus du modèle du "métrosexuel", les personnages féminins gagnent de l'indépendance et de l'assurance. A l'image des exemples de fictions cités cidessus, bon nombre des dramas de ces dernières années ont ainsi pour personnage principal une femme. Les dramas en présentant également de nombreuses scènes de la ville moderne (rues, bars, restaurants, hôtels, transports en commun, aéroports, parcs...) où les personnages, jeunes et beaux se déplacent et communiquent aisément grâce à la technologie (automobile, télécommunication), semblent faire l'apologie de la vie urbaine. Depuis le début des années 2000, de plus en plus de tournages ont lieu à l'étranger permettant aux téléspectateurs de s'échapper un instant mais aussi pouvoir mieux apprécier le confort de la ville sud-coréenne. La qualité des images (norme HD), l'originalité des musiques, des dialogues, de la façon de filmer pour certains, mais aussi la place importance accordée à l'esthétique (beauté des acteurs et actrices, des paysages, des musiques) donnent aux dramas contemporains une touche néo-urbaine dans laquelle se retrouvent les jeunes Asiatiques. Cependant, en matière de tendance (trend), tout est 
éphémère. Le boom des fictions américaines et le renouveau des fictions japonaises en Corée le prouvent.

\section{BIBLIOGRAPHIE}

BENASSI, Stéphane (2000). Séries et feuilletons T.V : pour une typologie des fictions télévisuelles. Liège : Editions du Céfal (Collection Grand Ecran, Petit Ecran, essais). 192 p. ISBN 287130071-2.

CLEMENTS, Jonathan \& TAMAMURO, Motoko (2004). The Dorama encyclopedia : a Guide to Japanese TV Drama since1983. Stone Bridge Press. 448 p. ISBN-10 : 1880656817.

HONG-MERCIER, Seok-kyeong (2006). "La question de reconstitution dans le docu-fiction historique : Une hybridation entre la vraisemblance et l'authenticité". In Figures de l'art, "Les pouvoirs des images", Juin 2006.

\section{RÉSUMÉS}

A partir du début des années 90, la Corée du Sud, où les récits mélodramatiques sont particulièrement appréciés, s'est mise à produire des feuilletons télévisés d'après le modèle des trendy dramas japonais. Adoptées par le public, ces formes hybrides sont devenues des produits de consommation culturelle appréciés et ont été, à leur tour, récemment exportées en masse vers les autres pays asiatiques. La forme du trendy drama qui épouse parfaitement les genres du drame familial et du drame sentimental, se caractérise par un environnement urbain contemporain et de jeunes personnages en âge de se marier. Cette proximité dans le temps mais aussi dans l'espace avec les téléspectateurs permet de présenter toute une panoplie de produits placés et de faire de lui un diffuseur de tendances. Le Fusion Drama, né de l'adaptation du trendy drama au drame historique, est ainsi une forme plus courte et plus moderne des feuilletons historiques classiques. Il bénéficie, comme le trendy drama, d'une grande qualité esthétique, notamment dans la représentation des personnages, des paysages et de la musique. Bien que de nombreuses mini-séries sud-coréennes de type fusion ou trendy, présentent des structures narratives assez simples, l'utilisation de technologies audiovisuelles et de techniques de tournages innovantes associée à la recherche de concepts originaux et au recours à la fantaisie dans les musiques et les dialogues les rendent attirantes pour le public sud-coréen et asiatique.

\section{AUTEUR}

\section{STÉPHANE THÉVENET}

CEISM, Université Paris Sorbonne Nouvelle

Centre de recherche sur la Corée (EHESS / CNRS) 